\title{
ASYMPTOTIC EXPANSIONS FOR A REMARKABLE CLASS OF RANDOM WALKS
}

\author{
J. BOERSMA \\ Department of Mathematics, Eindhoven University of Technology, P.O. Box 513, 5600 MB Eindhoten, \\ The Netherlands \\ and \\ F.W. WIEGEL \\ Department of Applied Physics, Twente University of Technology, P.O. Box 217, 7500 AE Enschede, \\ The Netherlands
}

Received 27 May 1983

\begin{abstract}
This paper extends the research of Wiegel (J. Math. Phys. 21 (1980) 2111) on random walks which differ from free random walks through the occurrence of an extra weightfactor $(-1)$ at every crossing of a half-line. Starting from a new closed-form expression for the weight distribution of these walks, we derive various integral representations and asymptotic expansions for the total weight of all walks.
\end{abstract}

\section{Introduction and statement of the problem}

In a recent paper') one of us studied the properties of random walks in a plane which differ from free random walks through the occurrence of an extra weightfactor $(-1)$ at every crossing of some branchline $T$. The statistical distribution of these walks was derived in the case that $T$ is a half-line and the initial point of the walk is located on the continuation of $T$. Random walks of this type are remarkable because of their relation to (i) two-dimensional selfavoiding random walk $\mathrm{k}^{2.3}$ ); and (ii) the combinatorial solution of the Ising model and the free-fermion case of the eight-vertex model $^{4}{ }^{10}$ ).

The random walks studied in ref. 1 are of course equivalent to the paths of a Brownian particle, whereby the weight of a path is multiplied by the factor $(-1)$ at every crossing of the half-line $T$. In an accompanying paper $\left.{ }^{\prime \prime}\right)$ we present a closed-form expression for the weight distribution of such Brownian motion paths, valid for any fixed initial point of the paths. It is the aim of this paper to reconsider the problem of ref. 1 , and to extend the mathematical analysis by using the results of ref. 11 . In particular, various integral representations and asymptotic expansions are derived for the total weight of all walks.

We now turn to a more detailed statement of the problem. Consider an arbitrary random walk configuration $\mathrm{C}$ in a plane, which crosses the branchline $\mathrm{T}$ a number 
of times, denoted by $n(\mathrm{C})=0,1,2, \ldots$ Then the a priori weight $W(\mathrm{C})$ of the configuration $\mathrm{C}$ is defined by

$$
W(\mathrm{C})=(-1)^{n(\mathrm{C})} W_{0}(\mathrm{C}),
$$

where $W_{0}(\mathrm{C})$ is the a priori weight of the corresponding free random walk. We take $\mathrm{T}$ to be the half-line $\theta= \pm \pi, 0<r<\infty$, in terms of polar coordinates $r, \theta$ in the plane of the walks. We then introduce the weight distribution

$$
p(r, \theta, N)=\sum_{\mathrm{C}} W(\mathrm{C}),
$$

where the summation extends over all configurations $C$ with fixed initial point $\boldsymbol{r}_{0}=\left(r_{0}, \theta_{0}\right)$ and end point $\boldsymbol{r}=(r, \theta)$, which consist of $N$ steps, each of the same length $l$. In ref. 1 the function $p(r, \theta, N)$ has been evaluated asymptotically for very long walks, in the special case when $\theta_{0}=0$, with the result

$$
p(r, \theta, N)=2\left(\pi N l^{2}\right)^{-1} \exp \left[-\frac{r_{0}^{2}+r^{2}}{N l^{2}}\right] \sum_{n=0}^{\infty} I_{n+1 / 2}\left(\frac{2 r_{0} r}{N l^{2}}\right) \cos \left(n+\frac{1}{2}\right) \theta .
$$

Here $I_{v}$ denotes the modified Bessel function of order $v$.

By taking limits as $N \rightarrow \infty, l \rightarrow 0$, such that $N l^{2}=4 D t$ remains fixed, the random walks turn into the paths of a particle which performs a Brownian motion with diffusion coefficient $D$. These Brownian motion paths differ from free Brownian motion paths through an extra weightfactor $(-1)$ for every crossing of the half-line $\mathrm{T}$. The function $p(r, \theta, N)$ changes into $p(r, \theta, t)$, which is the total weight of all paths starting in $r_{0}$ at $t=0$ and reaching the end point $r$ at time $t$. In ref. 11 it was found that

$$
p(r, \theta, t)=(4 \pi D t)^{-1} \exp \left[-\frac{R^{2}}{4 D t}\right] \operatorname{erf}\left(\left(\frac{r_{0} r}{D t}\right)^{1 / 2} \cos \frac{1}{2}\left(\theta-\theta_{0}\right)\right),
$$

where

$$
R^{2}=r_{0}^{2}+r^{2}-2 r_{0} r \cos \left(\theta-\theta_{0}\right)
$$

is the square of the distance between $\boldsymbol{r}_{0}=\left(r_{0}, \theta_{0}\right)$ and $\boldsymbol{r}=(r, \theta)$. In (4), erf stands for the error function defined by (eq. 7.1.1 of ref. 12)

$$
\operatorname{erf}(z)=2 \pi^{-1 / 2} \int_{0}^{z} \mathrm{e}^{-t^{2}} \mathrm{~d} t
$$

The equivalence of the results (4) and (3), in the special case $\theta_{0}=0$, can easily be established by means of the identity for a series of modified Bessel functions, proved in the appendix of ref. 11. Furthermore, we quote from ref. 11 that 
$p(r, \theta, t)$ is a solution of the diffusion equation

$$
\frac{\partial p}{\partial t}=D \Delta p
$$

subject to the initial condition

$$
\lim _{t ! 0} p=\delta\left(\boldsymbol{r}-\boldsymbol{r}_{0}\right)
$$

here, $\Delta$ denotes the two-dimensional Laplace operator with respect to the coordinates $r, \theta$ of the end point.

By integration of $p(r, \theta, t)$ over the entire plane we obtain the total weight $W(t)$ of all paths at time $t$ (or equivalently, the total weight $W_{N}$ of all walks of $N$ steps), i.e.,

$$
W(t)=\int_{0}^{t} r \mathrm{~d} r \int_{-\pi}^{\pi} p(r, \theta, t) \mathrm{d} \theta .
$$

Clearly, $W(0)=1$ by (8). Notice also that for free Brownian motion paths $W(t) \equiv 1$. It will turn out that $W(t)$ depends on $t$ through the dimensionless parameter

$$
\lambda=r_{0}^{2} / 4 D t
$$

In section 2 we derive a variety of integral representations and series-expansions for the weight $W(t)$. These integral representations are used in section 3 to establish asymptotic expansions for $W(t)$, both for small and large time $t$. The results obtained considerably extend those of ref. 1 .

\section{Integral representations and series-expansions for $W(t)$}

Differentiate (9) with respect to $t$, then by means of (7) and Gauss' divergence theorem one has

$$
\begin{aligned}
W^{\prime}(t) & =D \int_{0}^{t} r \mathrm{~d} r \int_{-\pi}^{\pi} \Delta p(r, \theta, t) \mathrm{d} \theta \\
& =D \int_{0}^{x} \frac{1}{r}\left[\frac{\partial p}{\partial \theta}(r,+\pi, t)-\frac{\partial p}{\partial \theta}(r,-\pi, t)\right] \mathrm{d} r .
\end{aligned}
$$

The derivatives $\partial p / \partial \theta$ at $\theta= \pm \pi$ are determined from (4) and inserted into (11), 
yielding

$$
\begin{aligned}
W^{\prime}(t)= & -\frac{r_{0}^{1 / 2} \cos \theta_{0} / 2}{2 D^{1 / 2}(\pi t)^{3 / 2}} \int_{0}^{\infty} \exp \left[-\frac{\left(r_{0}+r\right)^{2}}{4 D t}\right] r^{-1 / 2} \mathrm{~d} r-\frac{r_{0} \sin \theta_{0}}{4 \pi D t^{2}} \\
& \times \int_{0}^{\infty} \exp \left[-\frac{r_{0}^{2}+r^{2}+2 r_{0} r \cos \theta_{0}}{4 D t}\right] \operatorname{erf}\left(\left(\frac{r_{0} r}{D t}\right)^{1 / 2} \sin \theta_{0} / 2\right) \mathrm{d} r .
\end{aligned}
$$

The latter result is integrated with respect to $t$, where we use the auxiliary integrals

$$
\begin{aligned}
& \int_{0}^{t} \exp \left(-a^{2} \tau^{-1}\right) \tau^{-3 / 2} \mathrm{~d} \tau=\frac{\pi^{1 / 2}}{a}\left[1-\operatorname{erf}\left(\frac{a}{t^{1 / 2}}\right)\right], \\
& \int_{0}^{t} \exp \left(-a^{2} \tau^{-1}\right) \operatorname{erf}\left(b \tau^{-1 / 2}\right) \tau^{-2} \mathrm{~d} \tau \\
& \quad=\frac{1}{a^{2}} \exp \left(-\frac{a^{2}}{t}\right) \operatorname{erf}\left(\frac{b}{t^{1 / 2}}\right)+\frac{b}{a^{2}\left(a^{2}+b^{2}\right)^{1 / 2}}\left[1-\operatorname{erf}\left(\frac{\left(a^{2}+b^{2}\right)^{1 / 2}}{t^{1 / 2}}\right)\right] .
\end{aligned}
$$

Thus by integration of (12), starting from the initial value $W(0)=1$, we obtain

$$
\begin{aligned}
W(t)= & 1+\int_{0}^{t} W^{\prime}(\tau) \mathrm{d} \tau \\
= & 1-\frac{\cos \theta_{0} / 2}{\pi} \int_{0}^{\infty}\left[1-\operatorname{erf}\left(\lambda^{1 / 2}(1+s)\right)\right] \frac{s^{-1 / 2}(1+s) \mathrm{d} s}{1+s^{2}+2 s \cos \theta_{0}} \\
& -\frac{\sin \theta_{0}}{\pi} \int_{0}^{\infty} \frac{\exp \left[-\lambda\left(1+s^{2}+2 s \cos \theta_{0}\right)\right]}{1+s^{2}+2 s \cos \theta_{0}} \operatorname{erf}\left(2(\lambda s)^{1 / 2} \sin \theta_{0} / 2\right) \mathrm{d} s,
\end{aligned}
$$

where the original integrals have been simplified by the substitution $r=r_{0} s$, and $\lambda$ is given by (10). The integral representation (14) can be further reduced to

$$
\begin{aligned}
W(t)= & \frac{\cos \theta_{0} / 2}{\pi} \int_{0}^{\infty} \operatorname{erf}\left(\lambda^{1 / 2}(1+s)\right) \frac{s^{-1 / 2}(1+s) \mathrm{d} s}{1+s^{2}+2 s \cos \theta_{0}} \\
& -\frac{\sin \theta_{0}}{\pi} \int_{0}^{\infty} \frac{\exp \left[-\lambda\left(1+s^{2}+2 s \cos \theta_{0}\right)\right]}{1+s^{2}+2 s \cos \theta_{0}} \operatorname{erf}\left(2(\lambda s)^{1 / 2} \sin \theta_{0} / 2\right) \mathrm{d} s .
\end{aligned}
$$

Notice that both integrals in (15) vanish when $\lambda=0$, hence, $W(t) \rightarrow 0$ as $t \rightarrow \infty$. 
Consider now the special case $\theta_{0}=0$, previously treated in ref. 1 . Then the integral representation (15) simplifies to

$$
\begin{aligned}
W(t) & =\frac{1}{\pi} \int_{0}^{x} \operatorname{erf}\left(\lambda^{1 / 2}(1+s)\right) \frac{s^{-1 / 2} \mathrm{~d} s}{1+s} \\
& =\frac{1}{\pi} \int_{1}^{\infty} \operatorname{erf}\left(\lambda^{1 / 2} \sigma\right) \frac{\mathrm{d} \sigma}{\sigma(\sigma-1)^{1 / 2}} .
\end{aligned}
$$

In this case the representation (12) for $W^{\prime}(t)$ can be reduced to

$$
W^{\prime}(t)=-\frac{r_{0}^{1 / 2}}{D^{1 / 2}(\pi t)^{3 / 2}} \int_{0}^{x} \exp \left[-\frac{\left(r_{0}+\rho^{2}\right)^{2}}{4 D t}\right] \mathrm{d} \rho
$$

The latter integral can be evaluated in terms of Hardy's function $\operatorname{Ei}_{4}\left(\lambda^{1 / 2} / 2\right)$, which in its turn is expressible in terms of the modified Bessel function $K_{1 / 4}(\lambda / 2)$; cf. eqs. $10.2(3), 10.21(3)$ of ref. 13. Thus we find

$$
W^{\prime}(t)=-\frac{r_{0}}{D^{1 / 2}(2 \pi t)^{3 / 2}} \exp \left[-\frac{r_{0}^{2}}{8 D t}\right] K_{1 / 4}\left(\frac{r_{0}^{2}}{8 D t}\right)
$$

The present expression for $W^{\prime}(t)$ is integrated with respect to $t$, starting at $t=0$ where $W(0)=1$. By applying the substitution $r_{0}^{2} / 8 D t=s$, we obtain the integral representation

$$
W(t)=1-\frac{1}{\pi^{3 / 2}} \int_{i / 2}^{x} \mathrm{e}^{-s} K_{1 / 4}(s) s^{-1 / 2} \mathrm{~d} s
$$

or equivalently, by eq. 13.21(7) of ref. 13,

$$
W(t)=\frac{1}{\pi^{3 / 2}} \int_{0}^{i / 2} \mathrm{e}^{-s} K_{1 / 4}(s) s^{-1 / 2} \mathrm{~d} s
$$

valid for $\theta_{0}=0$, where $\lambda$ is given by $(10)$.

From eq. (12) of ref. 11 we quote the representation

$$
p(r, \theta, t)=(2 \pi D t)^{-1} \exp \left[-\frac{r_{0}^{2}+r^{2}}{4 D t}\right] \sum_{m=0}^{\infty} I_{m+1 ; 2}\left(\frac{r_{0} r}{2 D t}\right) \cos \left(m+\frac{1}{2}\right)\left(\theta-\theta_{0}\right)
$$

by a series of modified Bessel functions. In the special case $\theta_{0}=0,(21)$ reduces to (3). The expansion (2l) is inserted into the integral (9), then the $\theta$-integration is trivial. As for the $r$-integration, we first integrate by parts whereupon the 
resulting integral is determined by means of eqs. 9.6 .26 and 11.4 .31 of ref. 12 . In this manner we obtain the series-expansion

$$
\begin{aligned}
W(t)= & \pi^{-1 / 2} \lambda^{1 / 2} \mathrm{e}^{-\lambda / 2} \sum_{m=0}^{\infty}(-1)^{m}\left(m+\frac{1}{2}\right)^{-1} \\
& \times\left[I_{m / 2-1 / 4}\left(\frac{\lambda}{2}\right)+I_{m / 2+3 / 4}\left(\frac{\lambda}{2}\right)\right] \cos \left(m+\frac{1}{2}\right) \theta_{0},
\end{aligned}
$$

where $\lambda$ is given by (10). The latter series converges for any $\lambda>0$, however, the expansion is useful especially for small values of $\lambda$.

In the special case $\theta_{0}=0,(22)$ reduces to eq. (39) of ref. 1 . In this case we derive yet another expansion for $W(t)$. We start from the integral representation (20) and express the integrand in terms of Kummer's function $M$, viz.

$$
\mathrm{e}^{-s} K_{1 / 4}(s)=2^{-3 / 4} \Gamma(1 / 4) s^{-1 / 4} M\left(\frac{1}{4}, \frac{1}{2},-2 s\right)-2^{3 / 4} \Gamma(3 / 4) s^{1 / 4} M\left(\frac{3}{4}, \frac{3}{2},-2 s\right)
$$

by successive use of eqs. 13.6.21, 13.1.3 and 13.1.27 of ref. 12. Then through a term-by-term integration we are led to the power-series expansion

$$
W(t)=2^{-1 / 2} \pi^{-3 / 2} \sum_{n=0}^{\infty} \frac{\Gamma(n / 2+1 / 4)}{n !\left(n+\frac{1}{2}\right)}(-1)^{e(n)}(4 \lambda)^{n / 2+1 / 4},
$$

where $e(n)$ is the largest integer $\leqslant(n+1) / 2$.

\section{Asymptotic expansions for $W(t)$}

Consider first the asymptotics of $W(t)$ for small values of $\lambda$, corresponding to large time $t$ by (10). From (22) and (24) we readily find the asymptotic expansions

$$
\begin{aligned}
W(t)= & \frac{2 \Gamma(1 / 4)}{\pi^{3 / 2}} \lambda^{1 / 4} \cos \theta_{0} / 2-\frac{4 \Gamma(3 / 4)}{3 \pi^{3 / 2}} \lambda^{3 / 4} \cos \left(3 \theta_{0} / 2\right) \\
& -\frac{\Gamma(1 / 4)}{15 \pi^{3 / 2}} \lambda^{5 / 4}\left\{5 \cos \theta_{0} / 2-2 \cos \left(5 \theta_{0} / 2\right)\right\}+\mathcal{O}\left(\lambda^{7 / 4}\right)
\end{aligned}
$$

and, in the special case $\theta_{0}=0$,

$$
W(t)=\frac{2 \Gamma(1 / 4)}{\pi^{3 / 2}} \lambda^{1 / 4}-\frac{4 \Gamma(3 / 4)}{3 \pi^{3 / 2}} \lambda^{3 / 4}-\frac{\Gamma(1 / 4)}{5 \pi^{3 / 2}} \lambda^{5 / 4}+\mathcal{O}\left(\lambda^{7 / 4}\right)
$$

truncated after three terms. The expansion (26) extends the result in eq. (40) of ref. 1.

Next we consider the asymptotics of $W(t)$ for large values of $\lambda$, corresponding to small time $t$. Since the weight $W(t)$ is an even function of $\theta_{0}$, it is sufficient to restrict the range for $\theta_{0}$ to $0 \leqslant \theta_{0} \leqslant \pi$. We first determine the asymptotic expansion 
of the derivative $\mathrm{d} W / \mathrm{d} \lambda$, for large values of $\lambda$. To that purpose we start from the integral representation (12) for $W^{\prime}(t)$, which is rewritten as

$$
\begin{aligned}
\frac{\mathrm{d} W}{\mathrm{~d} \lambda}= & -\frac{t}{\lambda} W^{\prime}(t) \\
= & \frac{\cos \theta_{0} / 2}{\pi^{3 / 2} \lambda^{1 / 2}} \int_{0}^{x} \exp \left[-\lambda(1+s)^{2}\right] s^{-1 / 2} \mathrm{~d} s \\
& +\frac{\sin \theta_{0}}{\pi} \int_{0}^{x} \exp \left[-\lambda\left(1+s^{2}+2 s \cos \theta_{0}\right)\right] \mathrm{d} s \\
& -\frac{\sin \theta_{0}}{\pi} \int_{0}^{r} \exp \left[-\lambda\left(1+s^{2}+2 s \cos \theta_{0}\right)\right]\left\{1-\operatorname{erf}\left(2(\lambda s)^{1 / 2} \sin \theta_{0} / 2\right)\right\} \mathrm{d} s
\end{aligned}
$$

where $\lambda$ is given by (10). The successive terms in (27) are shortly denoted by $T_{1}(\lambda)$. $T_{2}(\lambda), T_{3}(\lambda)$.

The first integral in (27) was met before in (17). Thus, by means of (18), $T_{1}(\lambda)$ can be expressed in terms of the Bessel function $K_{1 / 4}(\lambda / 2)$, viz.

$$
T_{1}(\lambda)=\frac{\cos \theta_{0} / 2}{2^{1 / 2} \pi^{3 / 2}} \lambda^{-1 / 2} \mathrm{e}^{-\lambda / 2} K_{1: 4}(\lambda / 2)
$$

For large values of $\lambda$, we replace $K_{14}(\lambda / 2)$ by its asymptotic expansion from eq. 9.7.2 of ref. 12 , which yields

$$
T_{1}(\lambda) \simeq \frac{\cos \theta_{0} / 2}{2^{1,2} \pi} \mathrm{e}^{-} ; \sum_{n=0}^{\infty} \frac{(1 / 4)_{n}(3 / 4)_{n}}{n !}(-1)^{n} \dot{\lambda}^{-n-1}
$$

as $\lambda \rightarrow \infty$; here the Pochhammer symbol $(a)_{n}$ is defined by

$$
(a)_{0}=1, \quad(a)_{n}=a(a+1) \ldots(a+n-1), \text { for } n=1,2,3, \ldots
$$

The second integral in (27) can be expressed in terms of an error function, thus leading to

$$
T_{2}(\lambda)=\frac{\sin \theta_{0}}{2 \pi^{1 / 2}} \lambda^{-1 / 2} \exp \left[-\lambda \sin ^{2} \theta_{0}\right]\left\{1-\operatorname{erf}\left(\lambda^{1 / 2} \cos \theta_{01}\right)\right\} .
$$

The asymptotic expansion of $T_{2}(\hat{\lambda})$ is determined by use of eq. 7.1 .23 of ref. 12, viz.

$$
\begin{aligned}
T_{2}(\lambda) \simeq & \pi^{-1 / 2} \sin \theta_{0} H\left(-\cos \theta_{0}\right) \lambda^{-1 / 2} \exp \left[-\lambda \sin ^{2} \theta_{0}\right] \\
& +\frac{\tan \theta_{0}}{2 \pi} \lambda^{-1} \mathrm{e}^{-\lambda} \sum_{n=1)}^{x}(-1)^{n}\left(\frac{1}{2}\right)_{n}\left(\lambda \cos ^{2} \theta_{0}\right)^{-n}
\end{aligned}
$$


as $\lambda \rightarrow \infty$; here, $H$ is the unit step function defined by $H(x)=1$ for $x>0$ and $H(x)=0$ for $x<0$. The expansion (32) is non-uniform in $\theta_{0}$, i.e., the expansion is valid for $\theta_{0}$ away from $\pi / 2$ such that $\lambda \cos ^{2} \theta_{0} \gg 1$. For $\theta_{0} \approx \pi / 2$ the original form (31) of $T_{2}(\lambda)$ should be retained.

In the third integral in (27) we apply the substitution $s \rightarrow \lambda^{-1} s$, then

$$
T_{3}(\lambda)=\frac{\sin \theta_{0}}{\pi} \lambda^{-1} \mathrm{e}^{-i} \int_{0}^{\infty} \mathrm{e}^{-s^{2} / \lambda} f(s) \mathrm{d} s,
$$

where

$$
f(s)=\exp \left[-2 s \cos \theta_{0}\right]\left\{1-\operatorname{erf}\left(2 s^{1 / 2} \sin \theta_{0} / 2\right)\right\} .
$$

The asymptotics of $T_{3}(\lambda)$ as $\lambda \rightarrow \infty$ is treated by the Mellin-transform technique of Bleistein and Handelsman ${ }^{14}$ ). This technique is based on Parseval's formula for Mellin transforms, generally given by

$$
\int_{0}^{\infty} h(\mu s) f(s) \mathrm{d} s=\frac{1}{2 \pi \mathrm{i}} \int_{c-\mathrm{i} \infty}^{c+i \infty} \mu^{-z} M[h ; z] M[f ; 1-z] \mathrm{d} z,
$$

where the integration path $\operatorname{Re}(z)=c$ should lie in the common strip of analyticity of the Mellin transforms $M[h ; z]$ and $M[f ; 1-z]$. These Mellin transforms are defined by

$$
M[h ; z]=\int_{0}^{\infty} s^{z-1} h(s) \mathrm{d} s,
$$

and likewise for $M[f ; 1-z]$. To use (35) in (33), we set $\mu=\lambda^{-1 / 2}, h(s)=\mathrm{e}^{-s^{2}}$, then

$$
M[h ; z]=\int_{0}^{\infty} s^{z-1} \mathrm{e}^{-s^{2}} \mathrm{~d} s=\frac{1}{2} \Gamma(z / 2)
$$

is analytic for $\operatorname{Re}(z)>0$, while $M[f ; 1-z]$ is analytic for $\operatorname{Re}(z)<1$. Thus the representation (33) passes into

$$
T_{3}(\lambda)=\frac{\sin \theta_{0}}{2 \pi} \lambda^{-1} \mathrm{e}^{-\lambda} \frac{1}{2 \pi \mathrm{i}} \int_{c-\mathrm{i} \infty}^{c+\mathrm{i} \infty} \lambda^{z / 2} \Gamma(z / 2) M[f ; 1-z] \mathrm{d} z,
$$

where $0<c<1$. The latter integrand is analytic for $\operatorname{Re}(z)<1$, except for simple poles at $z=-2 n, n=0,1,2, \ldots$. Following the technique of ref. 14, we displace the path of integration to the left. Then we obtain a representation by a series of 
residues which is precisely the asymptotic expansion of $T_{3}(\lambda)$ as $\lambda \rightarrow \infty$, viz.

$$
T_{3}(\lambda) \simeq \frac{\sin \theta_{0}}{\pi} \lambda^{-1} \mathrm{e}^{-\lambda} \sum_{n=0}^{\infty} \frac{(-1)^{n}}{n !} M[f ; 2 n+1] \lambda^{-n} .
$$

The coefficients $M[f ; 2 n+1]$ in (39) can be evaluated through integration by parts, for example

$$
\begin{aligned}
M[f ; 1] & =\int_{0}^{x} \exp \left[-2 s \cos \theta_{0}\right]\left\{1-\operatorname{erf}\left(2 s^{1 / 2} \sin \theta_{0} / 2\right)\right\} \mathrm{d} s \\
& =\frac{1}{2 \cos \theta_{0}}-\frac{\sin \theta_{0} / 2}{\pi^{1 / 2} \cos \theta_{0}} \int_{0} \mathrm{e}^{-2 s} s^{-1 / 2} \mathrm{~d} s \\
& =\frac{1-2^{1 / 2} \sin \theta_{0} / 2}{2 \cos \theta_{0}}=\frac{1}{2\left(1+2^{1 / 2} \sin \theta_{0} / 2\right)}
\end{aligned}
$$

and similarly

$$
M[f ; 3]=\frac{8+9 \sqrt{2} \sin \theta_{0} / 2+6 \sin ^{2} \theta_{0} / 2}{32\left(1+2^{1 / 2} \sin \theta_{0} / 2\right)^{3}} .
$$

By combining (31) and the leading terms of the expansions (29) and (39), we obtain the asymptotic expansion of the derivative $\mathrm{d} W / \mathrm{d} \lambda$, viz.

$$
\begin{aligned}
\frac{\mathrm{d} W}{\mathrm{~d} \lambda}= & \frac{\sin \theta_{0}}{2 \pi^{1 / 2}} \lambda^{-1 / 2} \exp \left[-i \sin ^{2} \theta_{0}\right]\left\{1-\operatorname{erf}\left(\lambda^{1 / 2} \cos \theta_{0}\right)\right\} \\
& +\frac{1}{2^{1 / 2} \pi} \frac{\cos \theta_{0} / 2}{1+2^{1 / 2} \sin \theta_{0} / 2} \dot{\lambda}^{-1} \mathrm{e}^{-\lambda}+\left(\left(\lambda^{-2} \mathrm{e}^{-\lambda}\right)\right.
\end{aligned}
$$

as $\lambda \rightarrow \infty$. If $\theta_{0}$ is away from $\pi / 2$, the error function in (42) may be replaced by its asymptotic expansion as in (32), thus leading to

$$
\begin{aligned}
\frac{\mathrm{d} W}{\mathrm{~d} \lambda}= & \pi^{-1 / 2} \sin \theta_{0} H\left(-\cos \theta_{0}\right) \lambda^{-1 / 2} \exp \left[-\lambda \sin ^{2} \theta_{0}\right] \\
& +\frac{1}{2^{1 / 2} \pi} \frac{\cos \theta_{0} / 2}{\cos \theta_{0}} \lambda^{-1} \mathrm{e}^{-\lambda}+\mathcal{C}\left(\lambda^{-2} \mathrm{e}^{-i}\right)
\end{aligned}
$$

as $\lambda \rightarrow \infty$. We shall use the latter expansion in the ranges $0 \leqslant \theta_{0} \leqslant \pi / 4$ and $3 \pi / 4 \leqslant \theta_{0} \leqslant \pi$, whereas in the range $\pi / 4 \leqslant \theta_{0} \leqslant 3 \pi / 4$ the form (42) is retained.

Finally, by use of the relation

$$
W(t)=1-\int_{\lambda}^{x} \mathrm{~d} W / \mathrm{d} \lambda \mathrm{d} \lambda
$$


we determine the asymptotic expansion of the weight $W(t)$ for large values of $\lambda$, corresponding to small time $t$. By integration of (43) we readily find the expansion

$$
\begin{aligned}
W(t)= & 1-H\left(-\cos \theta_{0}\right)\left\{1-\operatorname{erf}\left(\lambda^{1 / 2} \sin \theta_{0}\right)\right\} \\
& -\frac{1}{2^{1 / 2} \pi} \frac{\cos \theta_{0} / 2}{\cos \theta_{0}} \lambda^{-1} \mathrm{e}^{-\lambda}+\mathcal{O}\left(\lambda^{-2} \mathrm{e}^{-\lambda}\right)
\end{aligned}
$$

as $\lambda \rightarrow \infty$. The present asymptotic form is valid in the ranges $0 \leqslant \theta_{0} \leqslant \pi / 4$, where $H\left(-\cos \theta_{0}\right)=0$, and $3 \pi / 4 \leqslant \theta_{0} \leqslant \pi$, where $H\left(-\cos \theta_{0}\right)=1$. In the special case $\theta_{0}=0$ we have the expansion

$$
W(t)=1-\frac{\mathrm{e}^{-\lambda}}{2^{1 / 2} \pi}\left[\lambda^{-1}-\frac{19}{16} \lambda^{-2}+\mathcal{O}\left(\lambda^{-3}\right)\right]
$$

as $\lambda \rightarrow \infty$, obtained directly through integration of (29). For $\pi / 4 \leqslant \theta_{0} \leqslant 3 \pi / 4$ the asymptotic expansion of $W(t)$ is determined by integration of (42). Here we need the auxiliary result

$$
\begin{aligned}
& \int_{i}^{\infty} \lambda^{-1 / 2} \exp \left[-\lambda \sin ^{2} \theta_{0}\right]\left\{1-\operatorname{erf}\left(\lambda^{1 / 2} \cos \theta_{0}\right)\right\} \mathrm{d} \lambda \\
& =\frac{\pi^{1 / 2}}{\sin \theta_{0}}\left\{1-\operatorname{erf}\left(\lambda^{1 / 2} \sin \theta_{0}\right)\right\}\left\{1-\operatorname{erf}\left(\lambda^{1 / 2} \cos \theta_{0}\right)\right\} \\
& \quad-\cot \theta_{0} \int_{i}^{\infty} \lambda^{-1 / 2} \exp \left[-\lambda \cos ^{2} \theta_{0}\right]\left\{1-\operatorname{erf}\left(\lambda^{1 / 2} \sin \theta_{0}\right)\right\} \mathrm{d} \lambda,
\end{aligned}
$$

where in the latter integral the error function may be replaced by its asymptotic expansion. In this manner we find the expansion

$$
\begin{aligned}
W(t)= & 1-\frac{1}{2}\left\{1-\operatorname{erf}\left(\lambda^{1 / 2} \sin \theta_{0}\right)\right\}\left\{1-\operatorname{erf}\left(\lambda^{1 / 2} \cos \theta_{0}\right)\right\} \\
& +\frac{1}{2 \pi} \frac{\cos \theta_{0}-2^{1 / 2} \sin \theta_{0} / 2}{\sin \theta_{0}\left(1+2^{1 / 2} \sin \theta_{0} / 2\right)} \lambda^{-1} \mathrm{e}^{-\lambda}+\mathcal{O}\left(\lambda^{-2} \mathrm{e}^{-i}\right)
\end{aligned}
$$

as $\lambda \rightarrow \infty$, valid for $\pi / 4 \leqslant \theta_{0} \leqslant 3 \pi / 4$. In the special case $\theta_{0}=\pi / 2$, this expansion can be reduced to

$$
W(t)=1-\mathrm{e}^{-i}\left[\frac{1}{2} \pi^{-1 / 2} \lambda^{-1 / 2}+\frac{1}{4} \pi^{-1} \lambda^{-1}-\frac{1}{4} \pi^{-1 / 2} \lambda^{-3 / 2}+\mathcal{O}\left(\lambda^{-2}\right)\right]
$$

as $\lambda \rightarrow \infty$. Notice that the asymptotic forms of $W(t)$ are different in the two cases $\theta_{0}=\pi / 2$ and $\theta_{0}=0$. The asymptotic behavior of $W(t)$ as $\lambda \rightarrow \infty$, depends on $\theta_{0}$ in a non-uniform manner. 


\section{References}

1) F.W. Wiegel, J. Math. Phys. 21 (1980) 2111.

2) F.W. Wiegel, Physica 98A (1979) 345.

3) F.W. Wiegel, Fluid Flow through Porous Macromolecular Systems, Lecture Notes in Physics, vol. 121 (Springer, New York, 1980).

4) M. Kac and J.C. Ward, Phys. Rev. 88 (1952) 1332.

5) S. Sherman, J. Math. Phys. 1 (1960) 202.

6) S. Sherman, J. Math. Phys. 4 (1963) 1213.

7) P.N. Burgoyne, J. Math. Phys. 4 (1963) 1320.

8) N.V. Vdovichenko, Zh. Eksp. Teor. Fiz. 47 (1964) 715 [Sov. Phys. JETP 20 (1965) 477].

9) F.W. Wiegel, Phys. Lett. A41 (1972) 225.

10) F.W. Wiegel, Can. J. Phys. 53 (1975) 1148.

11) F.W. Wiegel and J. Boersma. Physica 122A (1983) 325.

12) M. Abramowitz and I.A. Stegun, Handbook of Mathematical Functions (Dover. New York. 1970).

13) G.N. Watson, A Treatise on the Theory of Bessel Functions, 2nd Edition (Cambridge Univ. Press, Cambridge, 1958).

14) N. Bleistein and R.A. Handelsman, Asymptotic Expansions of Integrals (Holt, Rinehart and Winston, New York, 1975) chap. 4. 